\title{
A NOTE ON REGULAR CURVES
}

\author{
LIDA K. BARRETT
}

A regular curve is a continuum such that for each point $p$ of the continuum there is an arbitrarily small open set containing $p$ with a finite boundary. If the continuum is compact this statement is equivalent to the statement that each pair of points can be separated by a finite number of points. A point is a regular point of a continuum if it is contained in arbitrarily small open sets with finite boundaries. Such a point is said to be of order $n$ if the boundary of each set consists of $n$ or fewer points, and $n$ is the smallest integer for which this is true. If $p$ is a regular point but no such integer $n$ exists then $p$ is said to be a point of increasing order (or of order $\omega$ ).

It is not true that if each pair of points of a continuum can be separated by $n$ points then each point is of order less than or equal to $n$. For example, any dendrite has the property that each pair of points can be separated by one point. However, this note will show that if each pair of points of a continuum can be separated by $n$ points, $n>2$, then the set of all points of order greater than $n$ must be countable, and that no one of the nonlocal separating points of order greater than $n$ is a limit point of nonlocal separating points of order greater than $n$. The proof will depend on three lemmas concerning cuttings that are perhaps of interest in themselves.

The fact that the local separating points of order greater than $n$ are countable follows from the well-known theorem of G. T. Whyburn (see [3, p. 61]) that the set of all local separating points of order greater than two of any locally compact separable metric space is countable. The theorem of this note gives conditions under which a set of nonlocal separating points is countable. That the nonlocal separating points of order $n$ need not be countable is shown by the examples given in [1] of regular curves of bounded order $n$ such that the nonlocal separating points of order $n$ are uncountable and form a perfect set. It should be noted that it is a direct consequence of Theorem 1.1 of [1] that if in a continuum every pair of points can be separated by $n$ points, then some set of fewer than $n$ points must separate the continuum.

Lemma 1. If $M$ is a compact continuous curve, $\left\{X_{i}\right\}$ a sequence of irreducible cuttings, $X$ the sequential limit of this sequence, then $X$ is either a cutting or a connected set.

Received by the editors September 6, 1960. 
Proof. Suppose $X$ is not a cutting and is not a connected set. Denote by $H$ and $K$ two mutually separated closed sets whose sum is $X$ and by $\epsilon$ a positive number less than half the distance from $H$ to $K$. Since $X$ does not separate $M$ there exists an open set $U$, containing $X$, each of whose points is contained within the distance $\epsilon$ from a point of $X$, which does not separate $M$. $U$ must be the sum of two mutually separated open sets $U_{1}$ and $U_{2}$, containing $H$ and $K$, respectively. Since $X$ is the sequential limit of the sequence of cuttings there exists an integer $t$ such that $X_{t}$ is contained in $U$, and intersects both $U_{1}$ and $U_{2} . M-X_{t}$ is the sum of two mutually separated sets $M_{1}$ and $M_{2}$. Since $M-U$ is connected, $M-U$ must be a subset of either $M_{1}$ or $M_{2}$, say $M_{1}$. Therefore, $M_{2}$ is a subset of $U . X_{t}$ is an irreducible cutting containing points of both $U_{1}$ and $U_{2}$ so both $U_{1}$ and $U_{2}$ must contain points of $M_{2}$. This means $U_{1} \cdot X_{t}$ separates $M$ and $X_{t}$ is not irreducible. This is a contradiction and the theorem is established.

COROLlary 1. If in addition to the hypothesis of Lemma 1 it is assumed that for each $i, X_{i}$ consists of less than $n$ points, then $X$ is either a cutting consisting of less than $n$ points or a single point.

Note that although each cutting of the sequence is an irreducible cutting the sequential limit need not be an irreducible cutting.

Lemma 2. If $M$ is a compact continuous curve, $\left\{X_{i}\right\}$ a sequence of cuttings, $X$ the sequential limit of the sequence, and there is a positive number $\epsilon$ such that for infinitely many $i, M-X_{i}$ is the sum of two mutually separated sets $H_{i}$ and $K_{i}$ each of diameter greater than $\epsilon$, then if the diameter of $X$ is less than $\epsilon, X$ is a cutting.

Proof. Suppose $X$ is not a cutting. Then there exists an open set $U$ of diameter less than $\epsilon$ containing $X$ whose complement is connected. There exists a positive integer $n$ such that for $i>n, X_{i}$ is a subset of $U . M-U$ is connected and, therefore for every $i>n$, must be a subset of either $H_{i}$ or $K_{i}$, say $H_{i}$. Therefore $K_{i}$ is a subset of $U$ and consequently of diameter less than $\epsilon$. This is a contradiction of the hypothesis of the lemma.

Corollary 2. If the set $X$ consists of a single point, then $X$ is a cut point.

For a related result concerning nonseparated cuttings see [2].

Any point of an irreducible cutting consisting of a finite number of points is a local separating point. Although in general it is not the case that any point of a finite cutting is a local separating point, the following is true. 
Leмma 3. If $M$ is a compact continuous curve, $n$ a positive integer, $\left\{X_{i}\right\}$ a sequence of irreducible cuttings of $M$ such that for each $i$, the number of points in $X_{i}$ is less than are equal to $n, X$ the sequential limit of this sequence, and $X$ is a cutting, then each point $x$ of $X$ is a local separating point.

Proof. Let $x$ be a point of $X$ and let $R$ be a connected open set containing $x$ such that some locally connected subcontinuum $J$ of $M$ contains $R$ and no point of $X$ distinct from $x$. For some integer $c$, for each $i>c$ there is a subset $X_{i}^{\prime}$ of $X_{i}$ which separates $J$. The sequential limit of the $X_{i}^{\prime}$ is $x$. Denote by $H_{i}$ and $K_{i}$ two mutually separated sets whose sum is $J-X_{i}^{\prime}$. For each $i$ both $H_{i}$ and $K_{i}$ must contain a point of the boundary of $R, F(R)$, since $X_{i}$ is an irreducible cutting. Let $2 \epsilon$ be the distance from $x$ to $F(R)$. For some integer $d$, for $i>d$ the maximum distance from $x$ to any point $p$ of $X_{i}^{\prime}$ is less than $\epsilon$ and therefore the diameters of $H_{i}$ and $K_{i}$ for $i>d$ are greater than $\epsilon$. The conditions of the hypothesis of Lemma 2 are therefore satisfied by $J,\left\{X_{i}^{\prime}\right\}$, and $H_{i}$, and $K_{i}$. Therefore, $x$ is a cut point of $R$ and, hence, a local separating point of $M$.

TheOREM. If $M$ is a compact continuous curve such that each pair of its points can be separated by $n$ points, $n>2$, then at most a countable number of the points of $M$ are of order greater than $n$.

Proof. Since all but a countable number of local separating points are of order two, the local separating points of order greater than $n$ are countable. Suppose that there are uncountably many nonlocal separating points of order greater than $n$. For each nonlocal separating point $p$ of order greater than $n$ let $\epsilon_{p}$ be a positive number such that there is no open set $U$ of diameter less than $\epsilon_{p}$ containing $p$ such that the number of points in the boundary of $U$ is less than or equal to $n$. Let $d$ denote a positive number such that for uncountably many points $p, \epsilon_{p}>d$ and let $T$ be the set of all such points. There is some point $t$ of $T$ which is a limit point of $T$. Let $\left\{t_{i}\right\}$ be a sequence of points of $T$ such that $t$ is the sequential limit of the sequence. Denote by $\left\{N_{i}\right\}$ a sequence of cuttings each consisting of $n$ points such that for each $i, N_{i}$ separates $M$ into two mutually separated sets $X_{i}$ and $Y_{i}$, where $X_{i}$ contains $t_{i}$ and $Y_{i}$ contains $t$. There exists some subsequence of the $N_{i}$, which for convenience denote again as $\left\{N_{i}\right\}$, such that $t$ belongs to the sequential limit $X$ of the sequence. Since $t$ is not a local separating point it follows from Lemma 3 that $X$ is not a cutting. Therefore, by Corollary $1, X$ must be a set whose only element is $t$. Since for all $i, X_{i}$ contains $t_{i}$ and $Y_{i}$ contains $t$, the diameters of 
$X_{i}$ and $Y_{i}$ are greater than $d$. Therefore, it follows from Lemma 2 that $t$ is a cut point. This is a contradiction and the proof is complete.

CoRollary. If $M$ is a compact continuous curve satisfying the hypothesis of the theorem and $T$ is the set of all nonlocal separating points of $M$ of order greater than $n$, then no point $t$ of $T$ is a limit point of $T$.

\section{BIBLIOGRAPHY}

1. Lida K. Barrett, Regular curves and regular points of finite order, Duke Math. J. vol. 22 (1955) pp. 295-304.

2. C. H. Harry, An examination of some cut sets of space, Bull. Amer. Math. Soc. vol. 38 (1932) pp. 427-433.

3. G. T. Whyburn, Analytic topology, Amer. Math. Soc. Colloquium Publications, vol. $28,1942$.

The University OF UtAH AND

The University of Wisconsin 